\title{
Intérêt et limites des implants courts
}

\section{RÉSUMÉ}

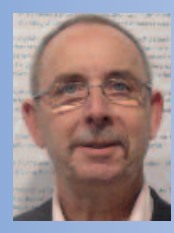

Olivier NÉDÉLEC

Académie nationale

de chirurgie dentaire,

DSO,

Docteur de l'Université Paris-Descartes,

Chirurgie orale et implantologie,

34, rue Laugier,

75017 Paris.
Les implants courts sont vendus par la plupart des firmes commerciales, encore faut-il que ce terme " court " soit défini. Pour notre part, ce sont des implants de $6 \mathrm{~mm}$ de long ou moins. Leurs indications sont limitées aux maxillaires postérieurs évitant ainsi des greffes sous-sinusiennes aux complications possibles, et des paresthésies du nerf alvéolaire inférieur. Leur mise en place ne diffère en rien des implants plus longs, encore faut-il leur assurer une parfaite stabilité primaire. Notre recul clinique est de 30 mois, il nous semble prudent d'attendre un peu avant d'étendre leurs indications.
- implants courts

- rugosité

- sinus maxillaire

- nerf alvéolaire inférieur

- Bio Management Complex ${ }^{\mathrm{TM}}$

- pilier Locator ${ }^{\mathrm{TM}}$
AOS 2011;253:55-61 


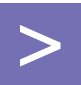

L'implantologie fait aujourd'hui partie de nos thérapeutiques au quotidien. Nos partenaires commerciaux rivalisent d'efforts pour proposer à la vente de nouveaux produits censés être toujours plus performants, aboutissant à des résultats esthétiques supérieurs ou bien à réduire le temps de traitement, notion qui importe souvent plus au praticien qu'au principal intéressé : le patient. Face à ces efforts de marketing développés au moment des grands congrès, il faut garder raison et ne pas être dupe face à une iconographie photographique et radiographique trop sélective. J'ai été, comme bien d'autres, confronté il y a quelques années aux conséquences des implants recouverts d'hydroxyapatite, " rien n'a été laissé au hasard » pouvions-nous lire dans les encarts publicitaires du distributeur de l'époque. De quoi devenir très méfiants et critiques ! Nous devons toujours rester maîtres de nos décisions thérapeutiques car elles engagent, il faut toujours le rappeler, notre responsabilité. Notre choix pour la nouveauté doit en particulier être dicté par l'expérience et les études de scientifiques dans des revues de qualité.

Le concept implantaire a connu de nombreuses modifications et évolutions ces dernières années, et parmi celles-ci les implants courts occupent une place de choix comme le confirme le nombre de communications sur ce thème dans les congrès nationaux et internationaux.

La définition des implants courts ne doit pas être source de confusions. Pour certains auteurs, il s'agit d'implants de 9 ou $10 \mathrm{~mm}$ de longueur. Pour notre part, ce sont des implants de 5 ou $6 \mathrm{~mm}$.

Ces implants courts sont vendus aujourd'hui par la plupart des firmes commerciales de par le monde. Ils permettent de repousser les limites de l'implantologie sans utiliser de greffes coûteuses, et souvent mal acceptées par les patients. Il s'agit du concept " graftless » [1] qui constitue une évolution significative de l'implantologie, et permet de réaliser une chirurgie moins invasive et sécurisée.

Des auteurs français [13] ont attiré l'attention de la communauté scientifique dès 2005 sur I'intérêt que pouvait apporter ce type de dispositif. Une conférence de consensus, sous l'égide de l'European Association for Osseointegration, a eu lieu en Suisse l'année suivante dont le thème était : I'influence de la longueur et du diamètre sur le taux de survie des implants. Les rédacteurs, qui étaient les mêmes auteurs, ont réalisé une revue Medline de 53 publications chez l'homme de 1990 à 2005, cependant, comme dit en introduction, les 34 publications sélectionnées faisaient référence à des implants de longueur variable [14]. Une étude rétrospective sur 408 implants courts en 2007 [7] confirmait l'intérêt de ce type de fixture avec un recul clinique important, les auteurs concluaient qu'à 5 ans les taux de succès étaient respectivement de 96,2 \% pour les implants de $7 \mathrm{~mm}$, et 97,1\% pour les implants de $8,5 \mathrm{~mm}$. Une autre publication de 2005 nous proposait déjà un guide d'utilisation de ces implants courts au regard des résultats cliniques [8].

Il faut rappeler que des implants courts de $5 \mathrm{~mm}$ étaient déjà commercialisés aux ÉtatsUnis : Bicon ${ }^{\mathrm{TM}}$ Dental Implants depuis les années 1990, et avait obtenu l'agrément FDA en 2002, et au Canada, Endopore ${ }^{\circledR}$ impacté, Innova (Sybron Dental Specialties) était une référence depuis les années 2000.

L'indication de ces implants est limitée à la réhabilitation des maxillaires postérieurs, qui 
constitue notre défi au quotidien. Pour rappel, après une extraction au maxillaire supérieur, la crête est soumise à une double résorption : la pneumatisation du sinus maxillaire en direction coronaire, et la résorption de la crête osseuse. La crête évolue apicalement en position palatine, cette résorption post-extractionnelle est centripète, contrairement à la mandibule où elle est centrifuge.

La pose d'implants de longueur standard dans ce faible volume osseux au maxillaire supérieur s'accompagne souvent de greffes sous-sinusales par voie crestale ou par abord latéral, greffes aux complications possibles, voire fréquentes pour des praticiens peu expérimentés : perforations et déchirures de la membrane de Schneider pouvant influer le taux de succès des

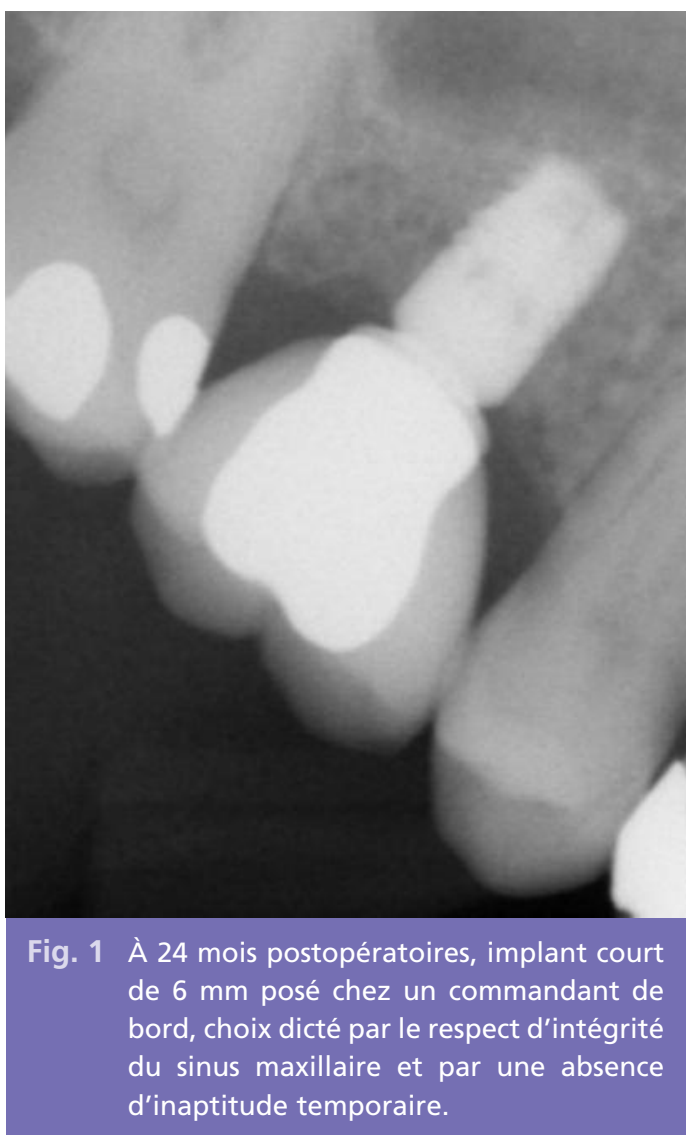

implants, paresthésies dues au paquet vasculonerveux infra-orbitaire, hémorragies de l'artère alvéolo-antrale, sinusites de confinement dues au blocage de l'ostium à 3 semaines... Ces complications ne sont pas anodines, elles doivent être anticipées, et peuvent même compromettre l'exercice d'une profession (fig. 1).

Quant à la mandibule, la proximité du nerf alvéolaire inférieur constitue un danger majeur dans les cas d'édentations anciennes (fig. 2).

Il est important de souligner aussi que ces zones maxillaires postérieures ainsi que les implants qui y sont posés sont soumis à des forces de mastication beaucoup plus importantes que dans la région antérieure, tout compromis chirurgical sera source d'échec.

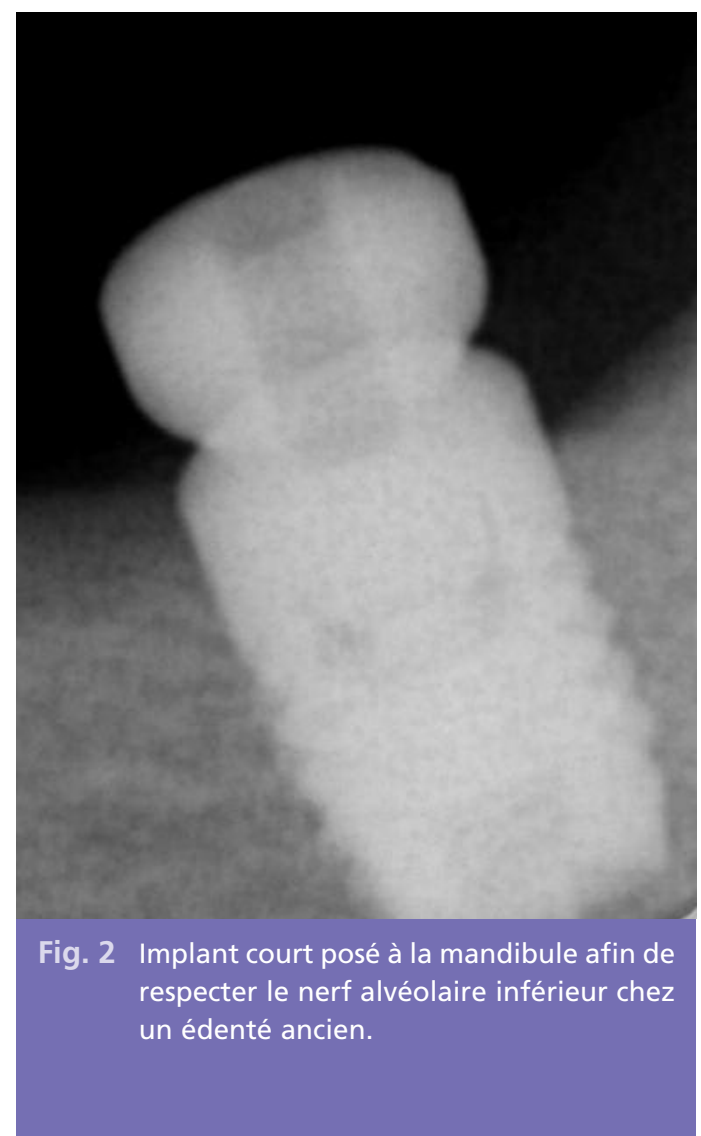


Des études déjà anciennes rapportaient un taux de succès inférieur pour les implants courts, mais elles concernaient des implants à surface usinée ayant un ancrage cortical et une faible participation de l'os spongieux [4-6, 17]. Dans deux études de 2006 [9, 15], les auteurs concluent que le taux de survie cumulatif des implants courts est comparable à celui des implants longs placés dans les mêmes conditions avec une technique chirurgicale correcte et des implants à surface rugueuse à condition d'obtenir une parfaite stabilité primaire.

La littérature nous apprend qu'il ne faut pas appliquer nos connaissances concernant le rapport couronne/racine des dents naturelles aux implants courts [16], cette étude basée sur

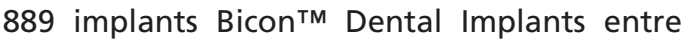
1992 et 2004 ne montre pas de différences de résultats quel que soit ce rapport avec un taux de survie de $98,2 \%$ (fig. 3 ).

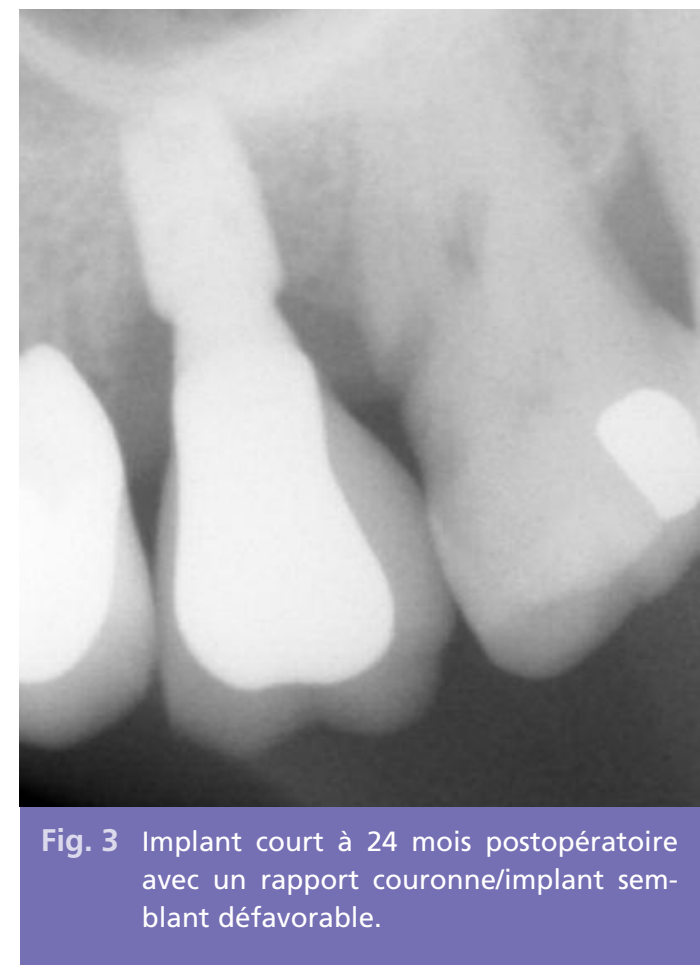

La longueur d'un implant n'est que I'une de ses caractéristiques. Son diamètre, sa forme (cylindrique, cylindro-conique, conique), son type d'intégration dans l'os (vissé, impacté), sa connexion, son état de surface sont des éléments qui doivent être pris en considération lors d'une décision chirurgicale implantaire [10]. Les effets interactifs de l'implant sur l'os crestal environnant sont en fait la résultante de tous ces facteurs, une étude de modèles par éléments finis [11] l'a bien expliqué, et les auteurs concluent que les implants étroits et courts devraient être évités dans un os de faible densité.

Un implant de plus gros diamètre permet de diminuer les contraintes appliquées, une analyse des contraintes par éléments finis [12] montre clairement qu'un implant court absorbe mieux les contraintes de cisaillement qu'un implant plus long car sa flexibilité per-

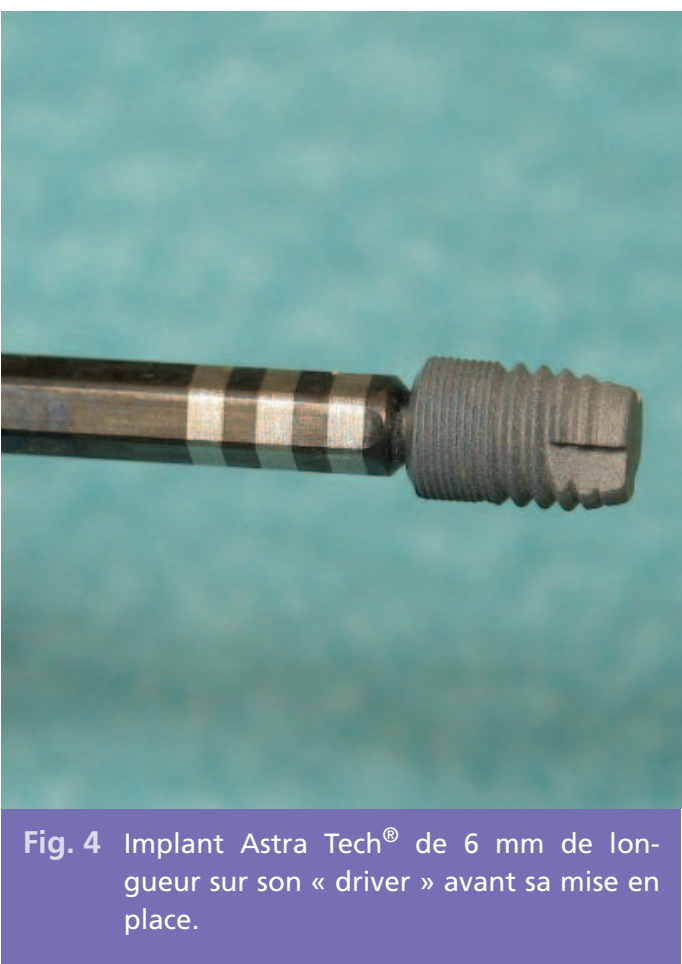


met d'amortir et d'absorber les contraintes avant l'arrivée de complications mécaniques et biologiques.

Parmi ces caractéristiques citées, une place prépondérante doit être faite à l'état de surface. Les implants que nous utilisons aujourd'hui sont sablés et/ou mordancés avec des acides forts (acide sulfurique, acide fluorhydrique, chlorure d'hydrogène), ce qui permet d'obtenir une intégration plus rapide. Nous pouvons probablement imaginer pour l'avenir des implants bioactifs associés à des facteurs de croissance. La surface rugueuse des implants aujourd'hui permet un ancrage à la fois cortical et spongieux, c'est dire qu'ils sont particulièrement bien indiqués dans I'os de type IV (classification de Lekholm et Zarb de 1985), rencontré dans la région maxillaire postérieure sous-sinusienne.

Notre expérience des implants courts concerne essentiellement, mais pas uniquement les implants Astra Tech ${ }^{\circledR}$ (Mölndal, Suède) commercialisés officiellement depuis le congrès mondial de Washington DC en 2008. Ces implants ne diffèrent en rien des autres implants de la firme suédoise (fig. 4), si ce n'est par leur longueur de $6 \mathrm{~mm}[2,3]$, leur diamètre est quant à lui de $4 \mathrm{~mm}$. À noter que cet implant, comme tous les autres de la gamme, existe désormais sous une forme à apex conique (tapered apex) destiné à un os de faible densité où un protocole de sous-forage peut être désiré.

Les implants Astra Tech ${ }^{\circledR}$ possèdent quatre caractéristiques fondamentales développées avec une approche biologique et biomécanique constituant le Bio Management Complex ${ }^{\mathrm{TM}}$ :

- la surface Osseospeed qui constitue une modification de la surface Tioblast ${ }^{\circledR}$ (surface de référence depuis 1990) par le fluorure à l'origine d'une plus forte différentiation ostéoblatique et une augmentation du contact os/implant in vivo ;

- le Conical Seal Design, jonction conique sans hiatus, qui transmet apicalement et repartit harmonieusement le pic des forces de cisaillement ;

- le microfiletage, Microthread, qui assure une distribution des charges, diminue les contraintes et stimule la formation osseuse conformément à la loi de Julius Wolff de 1892 ;

- le connective Contour ${ }^{\mathrm{TM}}$, optimisation de l'espace biologique bien avant le concept du Platform Switching.

D'un point de vue pratique, rien ne distingue la mise en place d'un implant court de celle d'un implant plus long. Cependant, la prudence s'impose car les forets à usage unique sans butée que nous utilisons arrivent très rapidement dans ce type d'os qui offre le plus souvent peu de résistance à cette longueur de $6 \mathrm{~mm}$. Les compétences chirurgicales acquises doivent jouer un rôle important afin d'acquérir la stabilité primaire de l'implant; dans un os de type IV nous optons, par sécurité, pour une période d'ostéointégration un peu allongée à quatre mois.

Dans l'état actuel de nos connaissances et étant donné l'absence de recul clinique suffisant (30 mois d'utilisation), nous limitons en ce qui nous concerne les indications des implants courts aux édentations unitaires et aux grandes reconstitutions en association avec des implants plus longs. Notre taux de succès cumulatif (CSRs des Anglo-Saxons) est de $98,47 \%$ : sur 65 implants posés, 35 au maxillaire, et 30 à la mandibule ; un implant unitaire sous-sinusien porteur de pilier Locator ${ }^{\mathrm{TM}}$ a été perdu récemment (stabilisation d'une prothèse adjointe complète). Au maxillaire, 13 implants sont des implants unitaires, et à la mandibule 9. Les autres implants sont supports encastrés de grandes reconstitutions comme dit ci-dessus en association avec 
des implants plus longs "standards". Ce taux de succès ne doit pas pour autant être extrapolé, il est plus que probable que nous retrouverons sur une série plus grande et avec plus de recul un taux de succès de 94 où $95 \%$, conforme à ce que nous apprend la bibliographie (l'implant perdu l'a été 27 mois après sa mise en place, mais on peut s'interroger afin de savoir si le pilier Locator ${ }^{\mathrm{TM}}$ est adapté à cet implant). Quant au niveau osseux, il est plus que probable que les clichés pris dans trois ans $(J+5)$ montreront une perte osseuse courante de l'ordre de $0,3 \mathrm{~mm}$.
L'intérêt des implants courts est donc primordial car ils permettent d'éviter des interventions pouvant entraîner une morbidité et une mortalité, ce concept évite les techniques chirurgicales lourdes de greffes sous-sinusiennes ou de transposition du nerf alvéolaire inférieur tout en tentant de répondre à toutes les situations cliniques. Néanmoins la prudence s'impose en l'état actuel de nos connaissances sur I'utilisation des implants courts pour des édentements unitaires, d'autant plus que les études sur les transferts de charge des piliers sont pratiquement inexistantes.

\section{Bibliographie}

1. Bedrossian $E$, Rangert $B$, Stumpel L, Indresano T. Immediate function with the zygomatic implant: a graftless solution for the patient with mild to advanced atrophy of the maxilla. Int J Oral Maxillofac Implants 2006;21(6):270-4.

2. Berglundh $\mathrm{T}$, Abrahamson I, Albouy JP, Lindhe J. Bone healing at implants with fluoride-modified surface: an experimental study in dogs. Clin Oral Implant Res 2007;18(2):147-52.

3. Ellingsen $\mathrm{JE}$, Johansson $\mathrm{CB}$, Wennenberg $A$, Holmén $A$. Improved retention and bone-to-implant contact with fluoride-modified titanium implants. Int J Oral Maxillofac Implants 2004;19(5):659-66.

4. Feldman $\mathrm{S}$, Boitel $\mathrm{N}$, Weng D, Kohles Sean S, Stach RM.

Five-Year Survival
Distributions of Short-Length (10 $\mathrm{mm}$ or less) Machined-Surfaces and Osseotite ${ }^{\circledR}$ Implants. Clin Implant Dent Relat Res April 2004;6(1):16-23.

5. Friberg $B$, Jemt $T$, Lekholm U.

Early failures in 4.641 consecutively placed Branemark dental implants: a study from stage 1 surgery to the connexion of completed prostheses. Int J Oral Maxillofac. Implants 1991;6(2):142-6.

6. Jaffin RA, Berman CL. The excessive loss of Branemark fixtures in type IV bone:

a 5-year analysis. J Periodontol 1991;62(1):2-4.

7. Malo $P$, de Araùjo Nobre $M$, Rangert $B$.

Shorts implants placed one-stage in maxillae and mandibles: a retrospective clinical study with 1 to 9 years of follow-up.

Clin Implant Dent Relat Res March 2007;9(1):15-21.

8. Misch CE.

Short dental implants:

a literature review and rationale for use.

Dent Today 2005;24(8):64-6,68.

9. Murray A.

Short dental implants as a treatment option: results from an observational study in a single private practice. JOMI sept-oct 2006; 21(5):769-76.

10. Nédélec $O$. Est-il raisonnable de caractériser un implant uniquement par sa longueur? Alpha Oméga News oct. 2008.

11. Petrie CS, Williams JL. Comparative evaluation of implant designs: influence of diameter, length, and taper on strains 
in the alveolar crest.

A three dimensional

finite-element analysis.

Clin Oral Implant Res

2005;16(4):486-94.

12. Pierrisnard $L$, Renouard $F$, Renault $\mathrm{P}$, Barquins $\mathrm{M}$. Influence of implant lenght and bicortical anchorage on implant stress distribution.

Clin Implant Dent Relat Res 2003;5(4):254-62.

13. Renouard F, Nisand D. Shorts implants in the severely resorbed maxilla: a 2-year retrospective clinical study.
Clin Implant Dent Relat Res 2005;7 Suppl 1: S104-10.

14. Renouard F, Nisand D. Impact of implant length and diameter on survival rates. First Consensus Conference, Feb 2006, Pfäffikon, Switzerland.

Clin Implant Dent Relat Res 2006;17 Suppl 2:35-51.

15. Romeo $E$, Ghisolfi $M$, Rozza R, Chiapasco M, Lops D.

Short $(8 \mathrm{~mm})$ dental implants in the rehabilitation of partial and complete edentulism: a 3-to 14-year longitudinal study. Int J Prosthodont 2006;19(6):586-92.

16. Schulte J, Flores AM, Weed M.

Crown-to-implant ratios

of single tooth implant-supported restorations.

J Prosthet Dent 2007, 98:1-5.

17. Tawil G, Younan R. Clinical evaluation of short, machined-surface implants followed for 12 to 92 months. Int J Oral Maxillofac Implants 2003;18(6):894-901.

\section{SUMMARY}

\section{Interest and limits of short implants}

Olivier NÉDÉLEC

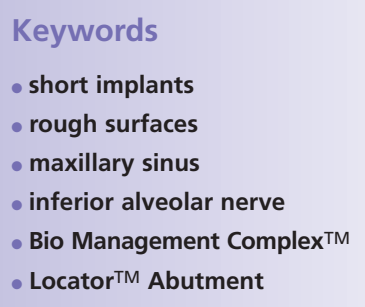

Short implants are commercialized today by the most part of the commercial companies; still it's necessary that this "short" term is defined. For our part, these are $6 \mathrm{~mm}$ length or less. Short implant placement in the posterior regions can be limited due to physical conditions: limited vertical bone height due to the expansion of the maxillary sinus or the proximity to the inferior alveolar nerve. Their installation is like for longer implants, still it's necessary to assure them a perfect primary stability. Our clinical outcome is of 30 months, it seems to us careful to wait a bit before spreading their indications. 

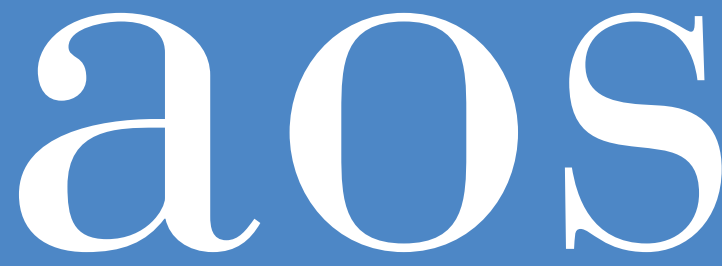

\section{Recommandations aux auteurs}

Les articles doivent être adressés en trois exemplaires au rédacteur-en-chef :

Dr Marc BERT, 1, rue de Chazelles - 75017 Paris.

Tout article doit être original et ne pas avoir déjà fait l'objet d'une publication.

Ils seront soumis au comité de rédaction des AOS qui, seul, acceptera, refusera ou demandera de modifier les textes qui lui sont proposés, en fonction de leur valeur scientifique ou clinique et de leur originalité.

Les auteurs préciseront leurs : nom, prénom, titres et affiliations universitaires ou hospitaliers éventuels, adresse, numéro de téléphone et de fax, ainsi que leur courriel.

\section{MANUSCRITS}

- dactylographier l'article en double interligne sur papier de format A4 (20 pages maximum) ;

- dactylographier les tableaux, légendes et références bibliographiques sur documents séparés ;

- le titre doit indiquer explicitement et avec le plus de concision possible le sujet du travail (60 signes, espaces compris) ; les intertitres ne doivent pas dépasser une demi-ligne ;

- joindre la traduction du titre de l'article en anglais ;

- joindre un résumé de 900 signes maximum (espaces compris) et sa traduction en anglais ;

- joindre 3 à 5 mots-clés choisis dans l'Index of Dental Litterature et sa traduction en anglais ;

- remettre trois exemplaires de la sortie laser ;

- joindre une copie en fichier informatique (texte enregistré de préférence sous Word 7).

\section{ICONOGRAPHIE}

- elle doit être originale et de bonne qualité. S'il s'agit d'une reproduction, une autorisation écrite de l'auteur de la photo, du schéma, de la radiographie, etc., doit être jointe avec les documents originaux;

- les schémas, dessins et radiographies doivent être réalisés à une échelle permettant une réduction ;

- chacune des illustrations doit comporter toutes les indications nécessaires (numéro de figure, nom du premier auteur, orientation). Les images en microscopie doivent comporter la mention du grossissement et l'échelle ;

- les figures doivent être numérotées et appelées par ce même numéro dans le texte ;

- les légendes des figures doivent être concises et figurer sur document séparé ;

- les documents iconographiques remis sous forme informatique sur CD-Rom devront être enregistrés sous Photoshop en JPG, EPS ou TIFF, résolution 300 dpi dans un format proche de celui souhaité dans la publication et être accompagnés de deux tirages papier en couleur.

\section{TABLEAUX}

- les présenter sur document séparé ;

- les légender ;

- les numéroter successivement en chiffres romains et les appeler dans le texte ;

\section{BIBLIOGRAPHIE}

Les références bibliographiques doivent être indexées dans le texte, présentées dans l'ordre de leur citation et nécessairement comporter les renseignements suivant l'ordre de la Convention dite de Vancouver :

\section{- pour un article de revue :}

Nom des auteurs, initiales des prénoms (Si plus de 6 auteurs, indiquer le premier suivi de et al.). Titre de la communication. Nom du journal (abrégé selon les normes internationales de I'Index Medicus, sans ponctuation après les abréviations) année;volume(numéro):pages (première et dernière pages).

Exemple: 1. Gary Bodo A, Merlier CH, Tarot P. Ostéosynthèse faciale par plaques miniaturisées à propos de 160 plaques du Pr Champy posées en un an. Ann Chir Plat Esthet 1983;28(1):41-47.

\section{- pour un article de livre :}

Même présentation des auteurs. Titre de l'article (dans la langue d'origine). In: noms et initiales des prénoms des "editors». Titre de l'ouvrage. Lieu de publication: nom de l'éditeur, année:pages première et dernière pages.

Exemple : 2. Bokos JC. Deposition structure and properties of pyrolitic carbon. In: Walker PL, ed. Chemistry and physics of carbon. New York : Marcel Dekern, 1972:70-81.

\section{- pour un livre:}

Même présentation des auteurs. Titre de l'ouvrage. Lieu de publication : nom de l'éditeur, année.

Exemple : 3. Garber D, Goldstein R. Inlays et onlays en céramique et en composite ; restaurations postérieures esthétiques. Paris : Éditions CdP, 1995.

La terminologie employée devra permettre une compréhension indiscutée des termes choisis. Référence pourra être faite aux divers ouvrages professionnels existants.

Vingt-cinq tirés-à-part de chaque article seront adressés aux auteurs après publication. Toute demande de tirés à part supplémentaires devra être formulée au moment du bon à tirer.

En aucun cas, les AOS ne pourront être tenues responsables pour la perte, le vol ou la détérioration des textes et documents reçus. 